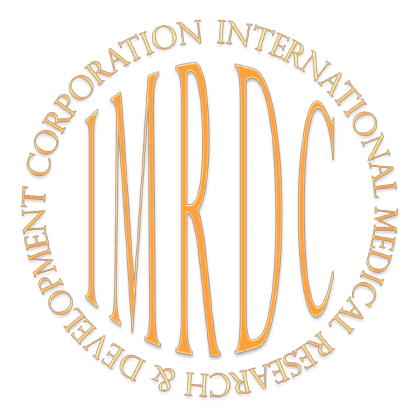

\title{
Fever as a Presentation of Tumoral Calcinosis: A Case Report
}

\author{
Nga V. Nguyen, MD*; Edgardo J. Guzman MD; \\ Christopher J. Mesa, MD; Shante A. Hinson, MD \\ Lincoln Medical and Mental Health Center \\ Bronx, NY, USA
}

\begin{abstract}
Tumoral calcinosis (TC) is a rare condition in which there is periarticular calcium deposition in the soft tissue forming a mass. The most common locations of TC are the larger joints such as the hip, shoulder, and knee, as well as the hands and wrists. Patients will often present with localized swelling, pain, and reduced joint mobility. We will discuss a 48-year-old male on hemodialysis who presented with a fever of unknown source and diffuse joint pain. He was found to have progressive, multiple tumor-like swellings on his shoulders, hands, and knees. He was diagnosed with TC and managed with a high dose phosphate binder with resolution of his fever and improvement in his pain.(International Journal of Biomedicine. 2018;8(1):75-78.)
\end{abstract}

Key Words: tumoral calcinosis $\bullet$ seronegative spondyloarthritis $\bullet$ hemodialysis $\bullet$ renal insufficiency

\section{Introduction}

Tumoral calcinosis (TC) is a rare condition and a complication of hemodialysis therapy. ${ }^{(1)}$ The lesions formed are described as a subcutaneous, periarticular, densely calcified mass confined to the soft tissue, generally at the extensor surface of the joint in the anatomic distribution of a bursa. The formation of the calcifications has been attributed to high calcium and phosphorus levels in the blood. The most common locations of TC are the larger joints such as the hip, shoulder, and knee, however, hands, wrists, and feet have been involved. (2) Plain radiographs reveal periarticular calcifications, sparing the underlying joint. ${ }^{(2)}$ If resected, the lesions have been described as cystic with a white to pale yellow chalky material, identified as calcium hydroxyapatite crystals with amorphous calcium carbonate and calcium phosphate. We report here a case of severe $\mathrm{TC}$ in a febrile patient.

\section{Case Presentation}

A 48-year-old male with a past medical history of human immunodeficiency virus on treatment, end stage renal disease on hemodialysis via permacath, hypertension, and chronic reactive arthritis/seronegative spondyloarthritis

*Corresponding author: Nga V. Nguyen, MD. Department of Internal Medicine, Lincoln Medical and Mental Health Center, Bronx, NY, USA.E-mail:ngavnguyen@gmail.com presented to the emergency department (ED) with complaint of fever, chills, malaise, and joint pain. The patient stated he had multiple joint pains, including his hands, shoulders and knees with his right knee being more prominent. In addition, he noticed "lumps" in several parts of his body growing in the past several months, causing discomfort and interfering with his activities of daily living. On review of systems, he denied any rashes, myalgia, sore throat, coughing, nausea, diarrhea, or genital discharge. Medications were consistent with his medical conditions. On examination, he was febrile at $101.5^{\circ} \mathrm{F}$, tachycardic with $\mathrm{HR}$ of $110 \mathrm{bpm}$, blood pressure was $100 / 60 \mathrm{mmHg}$, and pain was $10 / 10$ in severity. He was in mild distress with examination notable for tenderness and swelling on multiple joints including the knees and shoulders with the right knee being more prominent. His shoulders and knees had limited range of motion in all directions. In the ED, there was concern for septic arthritis, for which the patient had an arthrocentesis of his right knee. He was started empirically on cefepime and vancomycin. Of note, the patient had been admitted to an outside hospital two weeks prior for similar complaints and was treated with vancomycin at that time. Patient was advised to discontinue his Humira and prednisone. He was admitted to the hospital for further management of sepsis, likely secondary to septic arthritis.

During admission, blood tests: CD4 count of 277cells/ $\mathrm{mm}^{3}, \mathrm{WBC}-12.4 \times 10^{9} / \mathrm{L}, \mathrm{Hb}-8.7 \mathrm{~g} / \mathrm{dL}, \mathrm{ESR}-143 \mathrm{~mm} / \mathrm{hr}$, C-reactive protein $-28.89 \mathrm{mg} / \mathrm{dL}$, blood urea nitrogen $-62 \mathrm{mg} /$ $\mathrm{dL}$, creatinine $-10.3 \mathrm{mg} / \mathrm{dL}$, calcium $-9.2 \mathrm{mg} / \mathrm{dL}$, phosphate 
- $8.4 \mathrm{mg} / \mathrm{dL}$, parathyroid hormone - $389.5 \mathrm{pg} / \mathrm{mL}$, vitamin D - 31.5. Arthrocentesis of the right knee joint had fluid with a red/bloody appearance; decreased viscosity; WBC-0.9K/uL, 97\% segmented neutrophils; RBC/WBC clumps; negative for crystals. A right knee X-ray showed calcification on the medial and lateral collateral ligaments with no bony erosions (Fig.1).

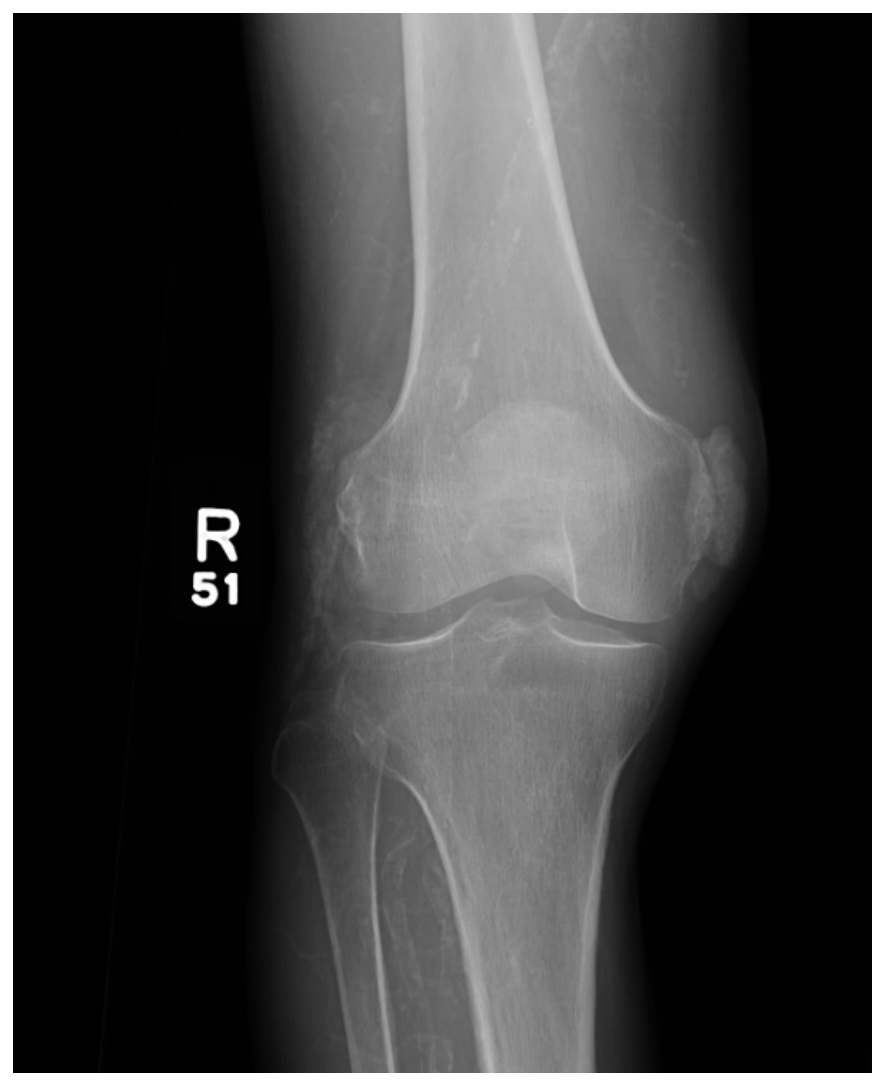

Fig. 1. Right knee X-ray on admission.

Given his persistent fever, there was an extensive investigation. Three blood cultures were negative along with a negative blood culture drawn from the permacath; fungal blood culture, urine culture, influenza swab, and chlamydia/gonorrhea urethral culture were also negative. During that time, he complained of a left shoulder mass which was causing discomfort. The impression was that the fever may be related to ongoing inflammation from joint pain. Recommendations were to obtain an echocardiogram to rule out culture-negative endocarditis and imaging of left shoulder, and to continue antibiotics as fever was trending downward, until a complete work-up was obtained. Rheumatology was consulted for a complaint of bilateral knee pain and bilateral hand pain, more on the right than the left. On examination at that time, the right hand had diffuse soft tissue swelling and tenderness over the second and third fingers, nodules on the left forearm, and a palpable mobile soft tissue mass on the left shoulder. There was also tenderness of the medial and lateral aspect of the right knee and tenderness of the lateral aspect of the left knee. The impression was TC, possibly due to secondary hyperparathyroidism in the setting of a hemodialysis patient. The recommendation was to obtain imaging of the left knee, left shoulder, and bilateral hands. It was also recommended to have a discussion with renal services to determine if the patient would benefit from a low calcium dialysate. Echocardiogram did not show vegetations, ruling out endocarditis. The left knee X-ray showed soft tissue calcifications over the lateral femoral condyle and in the anterolateral aspect of the knee joint. Hand X-rays showed multifocal calcifications in the hands and wrists (Fig. 2).

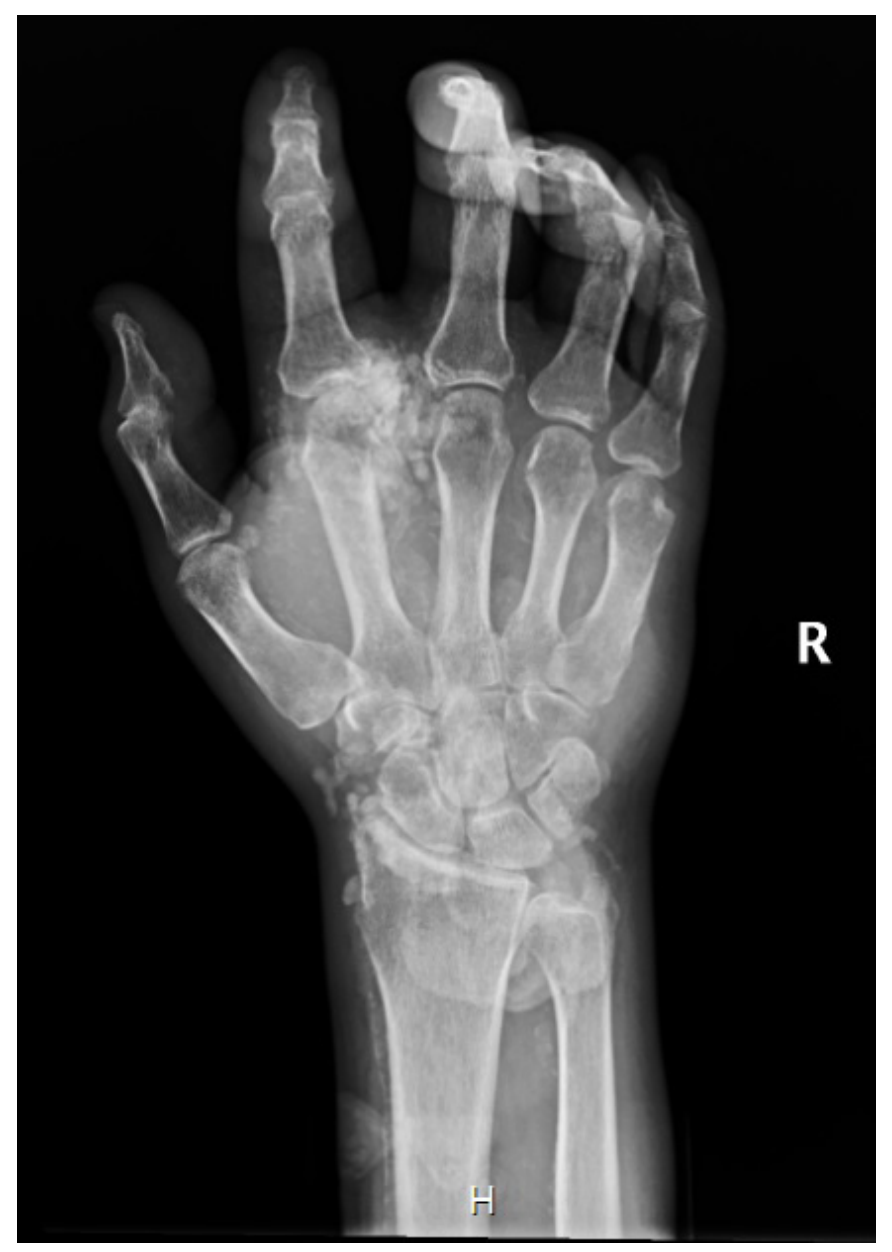

Fig. 2. Right hand X-ray during admission.

Shoulder X-rays showed calcification in the left shoulder joint greater than the right with no marginal erosions in the humeral heads (Fig. 3-4). After a multidisciplinary discussion, the patient was started on a phosphate binder, sevelamer $2400 \mathrm{mg}$ three times a day, a phosphate restricted diet, and low calcium dialysate during hemodialysis. He completed two weeks of cefepime and one week of vancomycin. As the patient was feeling well and fevers resolved, he was discharged to a short-term skilled nursing facility.

One month after his discharge from the hospital, the patient followed up in the rheumatology clinic. His symptoms improved; however, he was found to have an elevated calcium level of $12.5 \mathrm{mg} / \mathrm{dL}$ with improving phosphate levels. Given case reports of resolved calcium deposition with parathyroidectomy, he was referred to the endocrinology clinic, where it was discovered the patient was receiving calcitriol from his dialysis center. After calcitriol was discontinued, his calcium levels normalized. Three months after his discharge from the hospital, repeat X-ray imaging of his shoulders showed improving calcifications (Fig. 5-6). 


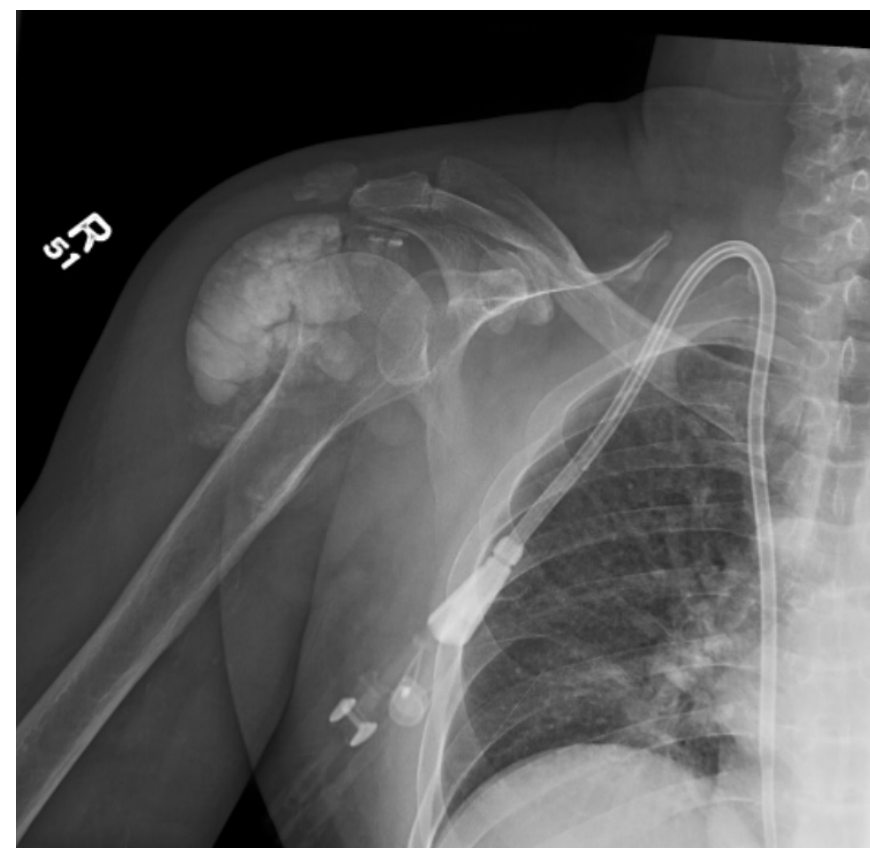

Fig. 3. Right shoulder X-ray during admission.

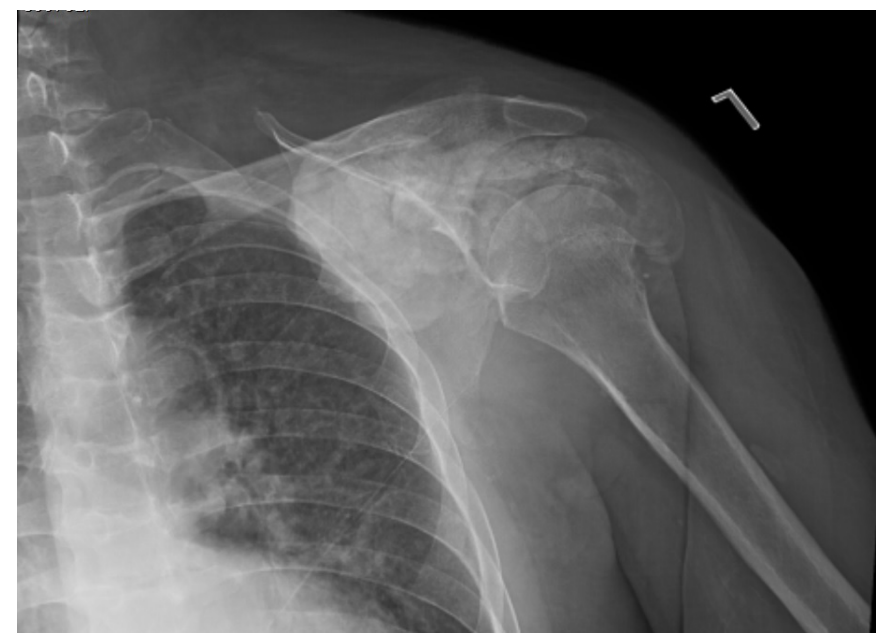

Fig. 4. Left shoulder X-ray during admission.

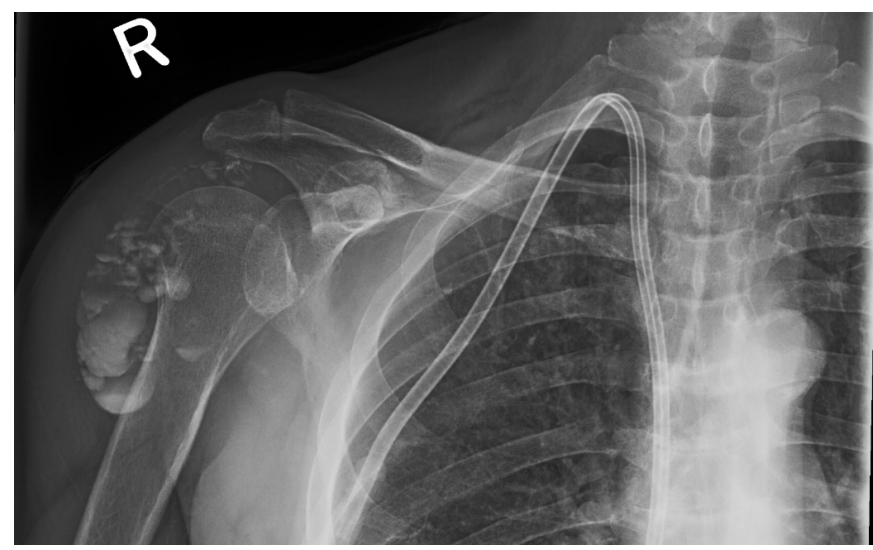

Fig. 5. Right shoulder X-ray 4 months after admission.

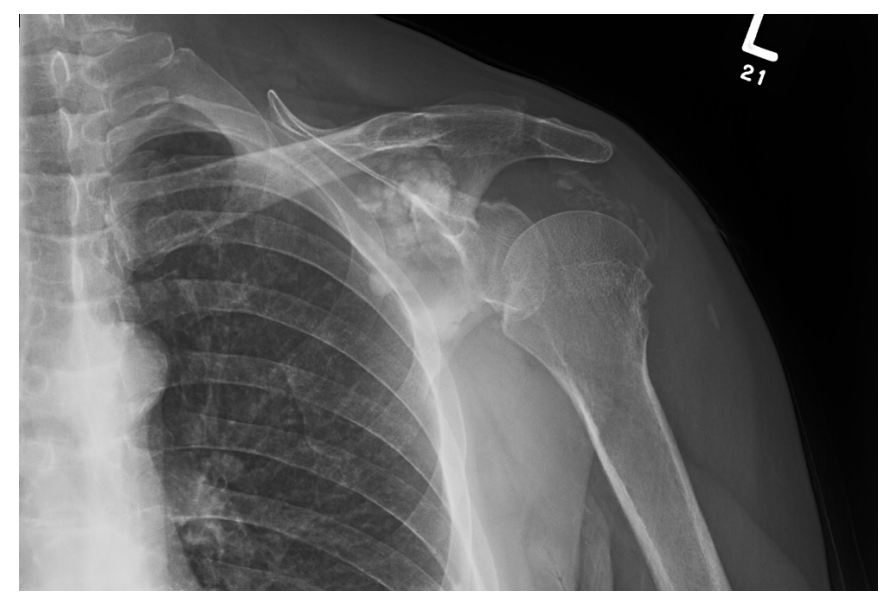

Fig. 6. Left shoulder X-ray 4 months after admission.

\section{Discussion}

TC is a rare condition in which a calcified lesion is deposited in the periarticular soft tissue, sparing the joint capsule. It can be found as a complication in those with end stage renal disease on hemodialysis due to high serum calcium or phosphate. In 1899, the original condition was first described by Duret in siblings who had multiple calcifications in the hips and elbows. ${ }^{(2)}$ In 1943, the term TC was first described by Inclan, who reported three separate cases with no familial relation. ${ }^{(3)}$ Initially, the condition was thought to be familial/hereditary as it was seen in young, healthy individuals. However, it can also be described as a secondary entity from chronic renal insufficiency, hyperparathyroidism, and hypervitaminosis ${ }^{(4)}$ The most common sites of tumoral calcinosis are the hip, shoulder, elbow, and knee. The TC deposition leads to reduced mobility, arthralgia, nerve compression, and pain. There are few reports of TC with presenting signs of systemic inflammation, such as fever and constitutional symptoms mimicking infections, as in this case.$^{(5)}$ Such a presentation could lead to a delay in management, inappropriate antibiotic use and extensive investigations. A multidisciplinary approach is often needed for the correct diagnosis and effective management.

Management of TC is often difficult and can be challenging. It involves dietary restriction of phosphate, noncalcemic phosphate binders, dialysis treatment with low calcium dialysate, parathyroidectomy in patients with high parathyroid hormone levels (due to tertiary hyperparathyroidism), surgical resection of the mass, or renal transplantation. ${ }^{(1)}$ In this case, dietary restriction of phosphate, a non-calcemic phosphate binder, and low calcium dialysate was used to manage the extensive TC. A few cases have provided immunohistochemical and microscopic findings from the resected soft tissue calcifications, indicating involvement of histiocytes and osteoclast-like giant cells of histiocyte origin. ${ }^{(6)}$ The fever and systemic inflammatory response from TC could be associated with the release of cytokines and anti-inflammatory markers from these cells. A few reports have described bisphosphate use to prevent a systemic inflammatory response due to cytokine release from osteoclastic activity. ${ }^{(5)}$ Ultimately, the selection 
of treatment depends on the severity of the presentation and a change in treatment if there is minimal or no response.

\section{Conclusion}

In our case, the patient presented with fever and pain from the diffuse periarticular soft tissue calcifications. Initially, it was presumed he had septic arthritis; however, the joint aspiration was not convincing. He continued to have fever despite being on antibiotics. After a multidisciplinary approach and extensive investigations, he was diagnosed with TC and started on the appropriate treatment with resolution of his fever and improvement in his symptoms. It is important to note that TC could present with pyrexia and systemic inflammatory symptoms/markers, which could delay management or lead to worsening complications if unrecognized.

\section{Competing interests} interests.

\section{References}

1. Binnani P, Aggarwal V, Bahadur M, Fulara N. Tumoral calcinosis (Teutschlander disease) in a dialysis patient. Indian J Nephrol. 2008;18(3):122-4. doi: 10.4103/0971-4065.43692.

2. Hammert WC, Lindsay LR. Tumoral calcinosis --or is it? A case report and review. Hand (NY). 2009; 4(2):119-22. doi: 10.1007/s11552-008-9132-0.

3. Inclan A. Tumoral calcinosis. JAMA. 1943;121:490-5. 4. Smack D, Norton SA, Fitzpatrick JE. Proposal for a pathogenesis-based classification of tumoral calcinosis. Int $\mathrm{J}$ Dermatol 1996;35(4):265-71.

5. Phanish MK, Kallarackal G, Ravanan R, Lawson TM, Baboolal K. Tumoral calcinosis associated with pyrexia and systemic inflammatory response in hemodialysis patients: successful treatment using intravenous pamidronate. Nephrol Dial Transplant. 2000;15(10):1691-3.

6. McGregor DH, Mowry M, Cherian R, McAnaw M, Poole E. Nonfamilial tumoral calcinosis associated with chronic renal failure and secondary hyperparathyroidism: report of two cases with clinicopathological, immunohistochemical, and electron microscopic findings. Hum Pathol. 1995; 26(6):607-13. 